\title{
COMPORTAMENTO DE MOVIMENTAÇÃO HORIZONTAL E VERTICAL DA TARTARUGA-DE-COURO Dermochelys coriacea
}

\author{
Milagros López-Mendilaharsu ${ }^{l, *}$ \& Carlos F.D. Rocha ${ }^{1}$ \\ ${ }^{1}$ Programa de Pós-Graduação em Ecologia e Evolução, Departamento de Ecologia, IBRAG, Universidade do Estado do Rio de Janeiro. Rua São Fran- \\ cisco Xavier 524, 20550-013, Maracanã, RJ, Brasil. \\ *Email: milagroslm@gmail.com
}

\section{RESUMO}

A biologia da tartaruga-de-couro (Dermochelys coriacea) é ainda relativamente pouco conhecida, fundamentalmente devido a seus hábitos de vida. D. coriacea é uma espécie altamente pelágica e cosmopolita que passa a maior parte da sua vida no oceano aberto, e que pode realizar migrações transoceânicas entre áreas de reprodução e de alimentação. Ao longo de sua extensa movimentação por porções oceânicas, a tartarugade-couro executa movimentos horizontais (ex. migrações) e verticais (ex. mergulhos) que constituem respostas comportamentais a diferentes fatores, como busca de ambiente apropriado para o crescimento, a alimentação e a reprodução, entre outros. No presente artigo, apresentamos uma revisão abrangente acerca do estado atual do conhecimento sobre os movimentos comportamentais horizontais e verticais realizados por $D$. coriacea, analisando como estes comportamentos são influenciados por diferentes fatores que regem a ecologia da espécie. Os estudos indicam que as feições oceanográficas constituem fatores-chave na determinação dos padrões de movimentação registrados para $D$. coriacea, principalmente durante as migrações e durante sua permanência em áreas de alimentação. Em geral a tartaruga-de-couro utiliza diferentes estratégias de navegação e dispersão nas diferentes regiões oceânicas, em alguns casos, os indivíduos da espécie se dispersam amplamente em varias direções e, em outros casos, seguem um padrão relativamente uniforme e persistente através do oceano. As tendências de movimentação durante o período de desova variam nas diferentes regiões oceânicas, influenciadas pela topografia do local e pela estratégia comportamental das fêmeas em relação aos custos energéticos durante a temporada reprodutiva (ex. comportamento de busca de alimento versus pouca atividade para reduzir o gasto metabólico).

Palavras-chave: Tartarugas marinhas, migrações, deslocamentos, duração do mergulho, profundidade do mergulho.

\section{ABSTRACT}

HORIZONTAL AND VERTICAL MOVEMENT BEHAVIOUR OF THE LEATHERBACK TURTLE Dermochelys coriacea. The general biology of the leatherback turtle (Dermochelys coriacea) still remains poorly understood, mainly because of its life-history characteristics. $D$. coriacea is a highly pelagic cosmopolitan species that spends most of its life in the open sea, and is able to perform transoceanic migrations from reproductive to foraging zones and back. Over their long travels throughout the sea, the leatherback turtle moves both horizontally (e.g., migrations) and vertically (i.e. dives) in response to different factors, like searching for suitable habitats for growth, feeding and reproduction. The present article is a comprehensive review of the current knowledge about the horizontal and vertical behavioral movements performed by $D$. coriacea. An analysis of how these behaviors are influenced by different factors governing the ecology of this species is presented. Some studies indicate that oceanographic features are the key factors determining the movement patterns observed for $D$. coriacea, mainly during their geographic migrations and residence in feeding zones. In short, the leatherback turtle employs different navigation and dispersion strategies while in each different oceanic zone. On certain occasions, individuals may disperse in different directions while on other occasions they may follow a roughly uniform path across the sea. Movement trends during the nesting period vary between different oceanic zones depending on local topography and on the behavioral strategy adopted by the nesting females with respect to how to invest energy during the reproductive season (e.g., actively searching for prey versus limiting activity to reduce metabolic rate).

Keywords: Marine turtles, migrations, displacements, dive duration, dive depth. 


\section{RESUMEN}

\section{COMPORTAMIENTO DE MOVIMIENTO HORIZONTAL Y VERTICAL DE LA TORTUGA}

LAUD Dermochelys coriacea. La biología de la tortuga laúd (Dermochelys coriacea) aún es relativamente poco conocida, fundamentalmente debido a sus hábitos de vida. $D$. coriacea es una especie altamente pelágica y cosmopolita que pasa la mayor parte da su vida en el océano abierto, y que puede realizar migraciones transoceánicas entre áreas de reproducción y de alimentación. En sus extensos desplazamientos a través del océano, la tortuga de laúd ejecuta movimientos tanto horizontales (ej. migraciones) como verticales (ej. buceos) que constituyen respuestas de comportamiento a diferentes factores como búsqueda de un ambiente apropiado para el crecimiento, alimentación y reproducción, entre otros. En este trabajo, presentamos una amplia revisión acerca del estado actual del conocimiento de los movimientos horizontales y verticales realizados por $D$. coriacea, analizando como estos comportamientos son influenciados por diferentes factores que determinan la ecología de la especie. Los estudios indican que las características oceanográficas constituyen factores claves en la determinación de los patrones de movimiento registrados para $D$. coriacea, principalmente durante las migraciones y durante su permanencia en áreas de alimentación. En general la tortuga laúd utiliza diferentes estrategias de navegación y dispersión en las diferentes regiones del océano, en algunos casos, los individuos de la especie se dispersan ampliamente en varias direcciones y en otros casos siguen un patrón relativamente uniforme y persistente a través del océano. Las tendencias de movimiento durante el período de desove varían en las diferentes zonas oceánicas, influenciadas por la topografía del lugar y por la estrategia de comportamiento de las hembras en relación a los costos energéticos durante la temporada reproductiva (ej. comportamiento de búsqueda de alimento versus poca actividad para reducir el gasto metabólico).

Palabras clave: Tortugas marinas, migraciones, desplazamientos, duración del buceo, profundidad de inmersión.

\section{INTRODUÇÃO}

A tartaruga-de-couro, Dermochelys coriacea, constitui a única espécie da família Dermochelyidae, e difere consistentemente das demais espécies de tartarugas marinhas em alguns aspectos. Os indivíduos adultos de $D$. coriacea podem atingir tamanho corpóreo de mais de $2 \mathrm{~m}$ de comprimento do casco e pesar mais de $900 \mathrm{~kg}$, sendo o maior dos quelônios e um dos maiores répteis do mundo (Márquez 1990, Figura 1). As tartarugas-de-couro são os répteis de distribuição geográfica mais ampla e ocorrem ao longo de todos os oceanos tropicais, temperados e frios do mundo, desde o mar de Barents no hemisfério norte até a ilha Sul de Nova Zelândia no hemisfério sul (Carr 1952, Pritchard 1973, Márquez 1990).

Esta espécie apresenta uma redução extrema dos ossos da carapaça, que é composta por um mosaico de pequenas peças osteodérmicas poligonais sustentadas em uma matriz de tecido dérmico cartilaginoso. Possui sete quilhas longitudinais dorsais e cinco ventrais; as quilhas dorsais convergem posteriormente em uma base caudal arredondada, acima da cauda (Márquez 1990). Apresenta características anatômicas (ex. forma hidrodinâmica da carapaça, grandes nadadeiras anteriores, entre outras) e fisiológicas que as difere de outras espécies de tartarugas marinhas, especialmente pela possibilidade de controlar a temperatura (ex. mantendo a temperatura do corpo relativamente constante, tanto nas águas quentes dos trópicos ou frias das regiões temperadas) através de uma série de adaptações, tais como ajustes do fluxo sanguíneo, mecanismos do tipo "contra-corrente" de troca de calor no fluxo sanguíneo, isolamento periférico (ex. presença de tecido adiposo subepidérmico) e metabolismo muscular independente da temperatura (Wallace \& Jones 2008). Essas características e outras adaptações fisiológicas e cardiovasculares, por exemplo, uso de reservas de oxigênio no sangue disponíveis através de ajustes do fluxo sanguíneo, diminuição da freqüência cardíaca para conservar oxigênio durante mergulho ou aumento da mesma para facilitar o intercambio de gases em superfície (Lutcavage et al. 1992, Southwood et al.1999), permitem que esta espécie consiga sobreviver em ambientes com baixas temperaturas $\left(<10^{\circ} \mathrm{C}\right)$ (James \& Mrosovsky 2004) e mergulhar a grandes profundidades ( $>1000 \mathrm{~m})$ (Doyle et al. 2008).

A biologia de $D$. coriacea é ainda relativamente pouco conhecida, fundamentalmente devido a seus 


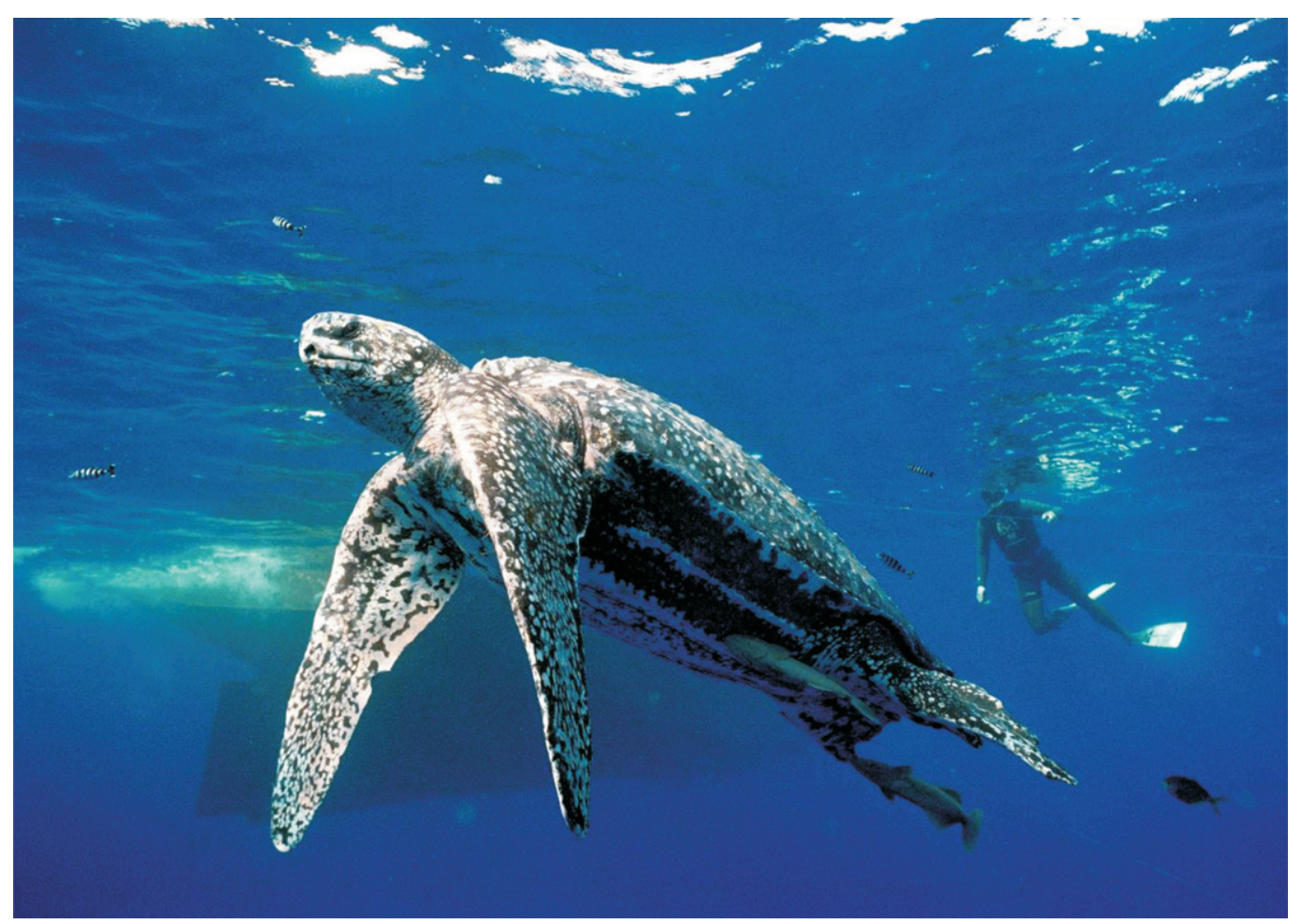

Figura1. Perspectiva de uma tartaruga-de-couro Dermochelys coriacea (Banco de Imagens Projeto Tamar).

Figure 1. Leatherback turtle Dermochelys coriacea (Courtesy of Projeto Tamar Image Data Bank).

hábitos de vida. São tartarugas altamente pelágicas e cosmopolitas que passam a maior parte da sua vida no oceano aberto e que podem realizar migrações transoceânicas entre áreas de reprodução e de alimentação (Benson et al. 2007a). Sua dieta consiste quase exclusivamente de zooplâncton gelatinoso (como medusas, sifonóforos e tunicados) (Davenport 1998, James \& Herman 2001, Witt et al. 2007).

O destino dos filhotes recém eclodidos dos ovos, após abandonarem as praias de desova, assim como quais são os habitats onde os jovens desta espécie ocorrem, ainda constituem grandes mistérios referentes à biologia desta espécie (Musick \& Limpus 1997). Alguns registros esporádicos sobre indivíduos jovens $(<100 \mathrm{~cm}$ de comprimento curvilíneo da carapaça - CCC) indicaram que eles geralmente permanecem em águas com temperaturas superiores a $26^{\circ} \mathrm{C}$ (Eckert 2002b).

Sabe-se que esta espécie migratória pode realizar movimentos trans-oceânicos através de milhares de quilômetros desde as praias tropicais de desova até as áreas temperadas de forrageio. Em escala global, as principais áreas de desova desta espécie se localizam no Oceano Atlântico, no nordeste da América do Sul (Guiana Francesa e Suriname) e no Gabão, África (Fosette et al. 2008a). No Atlântico ocidental, outras colônias menores estão situadas em ilhas do Caribe e na América Central, enquanto um número reduzido de fêmeas regularmente desova no litoral norte do estado do Espírito Santo, Brasil (Thomé et al. 2007). No Oceano Pacifico oriental as principais áreas de desova de $D$. coriacea se encontram no México e na Costa Rica (Eckert \& Sarti 1997, Reina et al. 2002), enquanto no Pacifico ocidental estão localizadas na Papua-Indonésia e na Papua-Nova Guiné (Benson et al. 2007b, Hitipew et al. 2007). No entanto, a situação desta espécie é critica em escala global. O forte declínio no número de fêmeas de $D$. coriacea em suas principais praias de desova no Oceano Pacífico nas últimas décadas (superior a $90 \% \mathrm{em}$ algumas colônias) (Spotila et al. 2000) é atribuído à morte acidental de indivíduos pela pesca industrial e artesanal e à intensa coleta de ovos nas praias de desova (Eckert \& Sarti 1997). Como resultado, as tartarugas-de-couro atualmente se encontram listadas como "criticamente em perigo" (IUCN 2007) e podem estar enfrentando um processo de iminente desaparecimento em algumas áreas do Oceano Pacífico (Spotila et al. 2000).

Esta espécie, que possui considerável vagilidade, caracteristicamente executa movimentos horizontais (migrações e deslocamentos) e verticais (mergulhos). 
Estes movimentos constituem respostas comportamentais a diferentes fatores, como busca de ambiente apropriado para o crescimento e escape a predadores, de áreas adequadas para obtenção de recursos alimentares e de sítios favoráveis ao acasalamento e à reprodução, entre outros. O termo "migração" refere-se ao movimento periódico ou sazonal do animal em resposta a fatores abióticos (ex. temperatura da água), à disponibilidade de alimento ou, ainda, para garantir a reprodução. No presente artigo, apresentamos uma revisão abrangente acerca do estado atual do conhecimento sobre os movimentos comportamentais horizontais e verticais realizados por $D$. coriacea, analisando como estes comportamentos são influenciados por diferentes fatores que regem a ecologia da espécie.

Ao realizar esta revisão nos restringimos aos artigos científicos publicados em renomados periódicos científicos, na última década (artigos no prelo, comunicações pessoais, textos monográficos e dissertações não foram utilizados). Os artigos analisados possuem variações nas metodologias utilizadas na obtenção dos dados. Por exemplo, o comportamento das fêmeas nas áreas de desova tem sido estudado através do uso de marcas eletrônicas (archival tags) as quais são capazes de coletar e armazenar dados comportamentais e ambientais (ex. Eckert et al. 1989, 1996, Southwood et al. 1999, 2005, Eckert 2002, Reina et al. 2005) e também mediante o uso de radiotransmissores (ex. Eckert 2002, Reina et al. 2005); os movimentos pós-desova têm sido conhecidos através do uso de placas metálicas convencionais (ex. Pritchard 1976) e através de telemetria por satélite (ex. Hughes et al. 1998, Luschi et al. 2003, Hitipew et al. 2007, Benson et al. 2007a). Neste último caso, com o surgimento de equipamentos capazes de coletar, armazenar e de transmitir dados de forma remota, também foi possível avançar no conhecimento sobre o comportamento vertical (mergulho) e horizontal (migração e deslocamento) nas diferentes fases do ciclo migratório de $D$. coriacea (ex. James et al. 2005b,c, James et al. 2006b, Doyle et al. 2008, Hays et al. 2006, Sale et al. 2006, Eckert 2006, Luschi et al. 2006). Embora tais estudos difiram quanto às abordagens e às metodologias utilizadas na obtenção dos dados, não temos razões para supor que as variações metodológicas possam prejudicar a qualidade das informações que os artigos fornecem.

\section{TIPOS DE MOVIMENTAÇÃO}

A habilidade de nadar e mergulhar eficientemente constitui uma característica central na sobrevivência de qualquer vertebrado marinho de respiração aérea. Principalmente para aqueles que vivem em um ambiente tridimensional sem refúgios e onde o alimento se encontra caracteristicamente distribuído em manchas (patches). Estes parâmetros do comportamento de movimentação são exemplos claros que permitem explicar o sucesso e a persistência com eficiência na vida aquática marinha por uma espécie como $D$. coriacea, um dos poucos répteis que apresenta hábitos de vida exclusivamente pelágicos.

Historicamente, a informação sobre a distribuição e os deslocamentos de $D$. coriacea esteve limitada aos dados de capturas acidentais obtidos a partir da indústria pesqueira, aos dados de recaptura de placas metálicas colocadas nas nadadeiras das tartarugas nas praias de desova e a observações episódicas no ambiente marinho (Pritchard 1973, 1976, Meylan 1982, Fretey 2001). Contudo, nas ultimas décadas, os avanços na tecnologia microeletrônica associada à telemetria por satélite têm permitido estudar o comportamento de movimentação deste quelônio em vida livre (Eckert 1998). Além da possibilidade de rastrear os movimentos e as migrações destes répteis, o desenvolvimento de equipamentos capazes de coletar, armazenar e transmitir dados de forma remota permitiu conhecer também o comportamento de mergulho de $D$. coriacea durante sua permanência em áreas de desova bem como durante suas migrações e permanência em áreas de alimentação (Hays et al. 2004b, Sale et al. 2006, James et al. 2006b).

\section{O USO DO AMBIENTE TRIDIMENSIONAL OCEÂNICO PELAS TARTARUGAS-DE-COURO: MOVIMENTOS VERTICAIS E HORIZONTAIS}

A compreensão dos padrões comportamentais de movimentação no oceano pelas tartarugas-de-couro tem sido intensivamente buscada nas últimas décadas e esses padrões podem ser considerados ainda pouco conhecidos (Eckert 2002a). Um ambiente como o oceânico, de natureza tridimensional, implica em complexas atividades que envolvem tanto movimentações horizontais quanto verticais, cada qual possivelmente determinada por funções específicas. 
Em termos deatividade de submersão, estudosnasáreas de desova com medidores de tempo e profundidade (TDRs - time depth recorders) permitiram conhecer alguns aspectos do comportamento de deslocamento vertical de $D$. coriacea, embora em uma janela de tempo relativamente limitada pela necessária recuperação dos equipamentos para tornar possível a obtenção dos dados. Com o tempo, o uso da telemetria por satélite permitiu incrementar o conhecimento sobre os deslocamentos e movimentações horizontais além das praias de desova. Inicialmente, devido às limitações tecnológicas dos aparelhos (tempo de vida da bateria, durabilidade do material implantado, entre outros), na maioria dos casos os trabalhos eram concluídos com uma simples descrição do ponto final da rota. Finalmente, após a incorporação de equipamentos com capacidade de armazenamento e de envio de dados via satélite (ex. profundidade e duração dos mergulhos, temperatura do ambiente, etc.) tem sido permitido avançar no conhecimento de como as tartarugas utilizam o ambiente tridimensional da coluna d’água nas diferentes regiões dos oceanos (Godley et al. 2007).

\section{MOVIMENTOS HORIZONTAIS}

Os padrões comportamentais e as características dos deslocamentos da tartaruga-de-couro variam ao longo do ciclo migratório; assim encontramos diferentes tipos de movimentação horizontal como os movimentos nas áreas de desova (ou movimentos internidais), os movimentos entre as áreas de desova/ reprodução e as áreas de alimentação e os movimentos em áreas de alimentação. Cada um deles possui particularidades comportamentais e possivelmente tem finalidades específicas que são decisivas para a aptidão dos indivíduos.

Os movimentos internidais são os movimentos realizados pelas fêmeas entre desovas consecutivas (às vezes percorrendo distâncias de dezenas até centenas de quilômetros) (Georges et al. 2007); os machos também se aproximam dessas áreas durante o período reprodutivo, mas nunca abandonam o mar (Miller 1997).

Os indivíduos após realizarem a atividade reprodutivatendem a migrarpara áreas mais produtivas em termos de recursos alimentares (Myers \& Hays 2006) com a finalidade de alimentação para reposição da energia e tecidos investidos na reprodução, para que um novo processo reprodutivo possa ser iniciado com eficiência (Broderick et al. 2001). Da mesma forma, após uma apropriada aquisição/reposição de recursos alimentares em áreas de alimentação e conseqüente reserva de energia. para subseqüente episódio de reprodução, a movimentação dos indivíduos tende a ocorrer das áreas de alimentação para as áreas de reprodução dando continuidade ao ciclo. Durante esses movimentos (ou migrações) os indivíduos podem percorrer distâncias de até milhares de quilômetros (Benson et al. 2007a). As fêmeas geralmente não desovam todos os anos, sendo que a maioria apresenta um intervalo de remigração (tempo entre sucessivas temporadas de desova) de 2-3 anos (Miller et al. 1997). Ainda assim, no Atlântico norte tem sido observado que alguns indivíduos costumam fazer migrações para regiões tropicais durante seus anos não reprodutivos (James et al. 2005b, 2006b). Para muitas espécies de tartarugas marinhas, as condições oceanográficas existentes nas áreas de alimentação antecedendo a temporada de desovas irão determinar a probabilidade de que ocorra nidificação (Broderick et al. 2001). Assim, diferenças na produtividade em determinadas regiões oceânicas afetam significativamente o intervalo de remigração das fêmeas (Saba et al. 2008, Caut et al. 2008).

Há ainda os movimentos que ocorrem enquanto os indivíduos se encontram nas áreas de alimentação, em áreas neríticas e/ou oceânicas com características oceanográficas favoráveis para a concentração de presas (James et al. 2005a,b, Eckert 2006).

A partir da velocidade de navegação, da trajetória e da forma das rotas e do tempo de permanência em certos locais é possível discriminar os tipos de movimentação horizontal (James et al. 2005b, Eckert 2006, Gaspar et al. 2006). Os movimentos migratórios entre áreas de reprodução e de alimentação (e viceversa) se caracterizamporvelocidades de deslocamento maiores que aquelas observadas durante o período reprodutivo ou durante a permanência em certas áreas de alimentação (James et al. 2005b,c, Eckert 2006). Por sua vez, a análise do trajeto e da forma da rota percorrida pelos indivíduos permite diferenciar comportamentos de viajem (movimento direto) e de alimentação (movimento errático) ao longo do ciclo migratório (Gaspar et al. 2006). Adicionalmente, o uso de determinadas técnicas para análise de séries 
temporais de localização espacial (state-space models) permite estimar parâmetros de movimento e modos comportamentais para definir os limites entre as diferentes fases do ciclo migratório (ex.período internidal, migrações e áreas de forrageamento) (Bailey et al. 2008, Jonsen et al. 2006).

Para animais marinhos que vivem e se deslocam em um meio em contínuo movimento, como é o oceano, os movimentos desse meio podem exercer consideráveis efeitos nas trajetórias dos indivíduos (Luschi et al. 2003, Lambardi et al. 2008). As áreas oceânicas visitadas pelas tartarugas-de-couro se caracterizam pela presença de correntes oceânicas e por feições oceanográficas prevalecentes que podem consistentemente afetar as trajetórias dos deslocamentos de duas formas: (1) diretamente, exercendo uma ação mecânica durante a movimentação, afetando a forma da rota; (2) indiretamente, a circulação oceânica é responsável pela distribuição espacial diferencial dos recursos alimentares no oceano, determinando a distribuição dos produtores (planctônicos) primários e consequentemente a dos níveis tróficos superiores incluindo os predadores de topo como as tartarugasde-couro (Lambardi et al. 2008).

\section{MOVIMENTOS VERTICAIS}

Como todos os vertebrados marinhos de respiração aérea, as atividades subaquáticas das tartarugas marinhas estão limitadas pela necessidade de repor as reservas de oxigênio. Um parâmetro fisiológico que permite fazer estimativas dos limites fisiológicos e energéticos das atividades é o limite aeróbico do mergulho ou aerobic dive limit (ADL), termo que se refere à duração do mergulho além do qual os níveis de lactato aumentam acima dos níveis de descanso (Kooyman et al. 1980). As tartarugas-de-couro apresentam adaptações fisiológicas cardiovasculares e respiratórias (tais como ajustes nos fluxos sanguíneos, bradicardia e grandes reservas de oxigênio nos músculos e no sangue) para realizar mergulhos profundos e prolongados (Lutcavage et al. 1992). Contudo, além dessas adaptações fisiológicas cardiovasculares e respiratórias, as necessidades metabólicas destes répteis marinhos também influenciam de forma significativa os investimentos de tempo nas diferentes atividades (Wallace \& Jones 2008).

Devido ao fato de que os movimentos das tarta- rugas-de-couro podem estar fortemente afetados por fatores oceanográficos, o comportamento de mergulho destas tartarugas pode apresentar variações espaciais e temporais, influenciados tanto por fatores ambientais como a temperatura da água, como pela disponibilidade local e a distribuição vertical das presas.

As tartarugas-de-couro apresentam padrões de mergulho distintos durante as diferentes atividades. Por exemplo, no mergulho subsuperficial $(<2-3 \mathrm{~m})$, as tartarugas utilizam esta profundidade de mergulho para percorrer maiores distâncias de forma mais eficiente, já que o mergulho é o suficientemente profundo para eliminar os efeitos de turbulência gerada pelo nado da tartaruga, mas ao mesmo tempo, raso o bastante para permitir uma rápida respiração quando necessário (Eckert 2002a, Reina et al. 2005); durante os mergulhos tipo- $\mathrm{U}$, as tartarugas diminuem sua atividade próximo ao fundo oceânico, onde descansam imóveis ou se movimentam de forma lenta; já os mergulhos tipo-V, caracterizados por um período de tempo $<1 \mathrm{~min}$ na profundidade máxima do mergulho, aparentemente possuem função de trânsito ou de busca (Reina et al. 2005).

\section{TENDÊNCIAS COMPORTAMENTAIS DURANTE AS MIGRAÇÕES E OS DESLOCAMENTOS GEOGRAFICOS}

Há diferentes tendências comportamentais realizadas durante os movimentos migratórios, que podem diferir dependendo se os movimentos migratórios são realizados nas áreas de desova, se realizados entre as áreas de desova e as áreas de alimentação, se realizados nas áreas de alimentação acompanhando manchas de produtividade, ou se realizados desde áreas de alimentação para áreas de reprodução após acumulo de energia para o processo reprodutivo. Cada um desses movimentos possui particularidades que são inerentes aos processos ecológicos e fisiológicos envolvidos. A seguir, detalhamos os comportamentos envolvidos em cada um desses movimentos migratórios.

\section{MOVIMENTOS NAS ÁREAS DE DESOVA (OU HABITATS INTERNIDAIS)}

As fêmeas adultas de forma geral retornam para as praias de desova em ciclos de aproximadamente 
2 a 3 anos. Durante a temporada reprodutiva elas costumam desovar nas praias em intervalos que variam entre 9 a 10 dias, chegando a colocar em média de 4 a 7 ninhadas (Miller 1997, Reina et al. 2002). Nesse período as fêmeas grávidas podem percorrer grandes distâncias (até centenas de quilômetros) entre eventos reprodutivos consecutivos, visitando águas jurisdicionais de diferentes países (Fossette et al. 2008b, Witt et al. 2008, Eckert 2006, Hitipeuw et al. 2007). As praias de desova das tartarugas-de-couro se localizam tanto em ilhas oceânicas, como em áreas continentais, onde os ambientes marinhos próximos às praias geralmente apresentam características físicas distintas. Em geral as tartarugas-de-couro viajam distâncias maiores entre uma desova e outra em regiões caracterizadas por extensas plataformas continentais (como no caso da Guiana Francesa ou Gabão) em comparação com as fêmeas que desovam nas ilhas do Caribe onde a plataforma peri-insular é muito estreita (Eckert 2002a, Georges et al. 2007). As diferenças observadas indicam a realização de mergulhos mais rasos com predominância de movimentos horizontais que são restritos em termos da profundidade pela batimetria local nas áreas de plataforma versus mergulhos mais profundos com deslocamentos menores no plano horizontal nas regiões onde as águas profundas ocorrem à pequena distância da costa (Eckert 2002a, George et al. 2007). As oportunidades de forrageamento geralmente são limitadas nas áreas de desova, onde as condições bióticas e abióticas diferem consistentemente em relação àquelas em geral encontradas nas áreas de alimentação (Eckert et al. 2006). Dessa forma, as tartarugas marinhas podem compensar os elevados custos energéticos (para a produção de ovos e nas atividades envolvidas no processo de oviposição) dispendidos durante a temporada reprodutiva de duas formas: (1) reduzindo a atividade e permanecendo em áreas restritas perto do local de desova, como foi observado para as fêmeas da região leste do Oceano Pacifico na Costa Rica (Reina et al. 2005); ou (2) mantendo uma atividade de busca e possível alimentação, como foi sugerido no caso de algumas colônias no Mar do Caribe e Oceano Atlântico tropical (Fossette et al. 2007, Georges \& Fossette 2006, Myers \& Hays 2006).

\section{MOVIMENTOS MIGRATÓRIOS ENTRE ÁREAS DE DESOVA E DE ALIMENTAÇÃO}

Os movimentos de migração entre áreas de desova e aquelas de alimentação em geral são caracterizados pormaiores velocidades denavegação que podem incluir extensos deslocamentos em águas oceânicas ou movimentos tanto costeiros como oceânicos com destino a habitats neríticos $(<$ 200m)de alimentação (Eckert et al. 2006, Eckert 2006, James et al. 2005b).

No Pacifico leste, após a temporada reprodutiva, as fêmeas que desovam nas praias da Costa Rica e do México seguem por um corredor migratório localizado ao sul da Costa Rica que atravessa a região equatorial passando pelas ilhas Galápagos rumo ao Pacifico sul (Morreale et. al. 1996, Eckert \& Sarti 1997, Shillinger et al. 2008). Durante esse percurso as tartarugas-de-couro atravessam fortes correntes na região equatorial que modelam a forma das rotas até seu destino final (Shillinger et al. 2008).

No entanto, algumas fêmeas que foram rastreadas desde colônias localizadas no Pacifico oeste (Indonésia e Nova Guiné) apresentaram diferentes comportamentos migratórios influenciados em parte pela época do ano em que ocorre a desova (verão boreal - Indonésia, ou verão austral - Nova Guiné), o que tenderá a determinar a realização de migrações para o hemisfério norte ou para o hemisfério sul (Hitipew et al. 2007, Benson et al. 2007a,b). As fêmeas que desovam no verão boreal nas praias da Indonésia têm mostrado que podem realizar migrações em diversas direções: rumo ao norte próximo do Japão, para o Pacífico leste (neste caso incluindo migrações trans-oceânicas até os Estados Unidos), ou rumo ao oeste permanecendo em águas tropicais perto da Malásia e Philipinas (Benson et al. 2007a). No caso da população que desova no verão austral, as fềmeas monitoradas mostraram um padrão consistente e relativamente uniforme de movimentação em direção sudeste rumo às águas do Pacífico sul (Benson et al. 2007b). Assim, foi sugerido que o destino final de tais migrações pode estar relacionado à disponibilidade de presas (na época adequada) nas diferentes regiões oceânicas (Benson et al. 2007a).

No Atlântico oeste, algumas fêmeas monitoradas desde as praias de desova localizadas na Guiana Francesa e Suriname (Ferraroli et al. 2004), Granada 
(Hays et al. 2004a), Trinidad (Eckert 2006) e Flórida, Estados Unidos (Eckert et al. 2006) se dispersaram amplamente através do Atlântico norte seguindo diversas rotas migratórias.

Já no Oceano Índico, logo após as fêmeas abandonarem as praias de desova na África do Sul, estas se mantiveram em ambientes oceânicos realizando movimentos ao longo do continente africano até o Oceano Atlântico, em provável associação com os fortes sistemas de correntes ali existentes (Lambardi et al. 2008).

\section{MOVIMENTOS EM ÁREAS DE ALIMENTAÇÃO}

$\mathrm{O}$ determinante primário dos movimentos e do comportamento das tartarugas-de-couro nas áreas de alimentação é a distribuição espacial e temporal da sua principal presa, o zooplâncton gelatinoso. No Atlântico norte ao longo da costa do Canadá, assim como no Pacifico leste, na costa de Oregon e da Califórnia, nos Estados Unidos, a ocorrência de tartarugas-de-couro têm sido frequentemente registrada nos meses da primavera e do verão e alimentando-se em hábitats neríticos, quando ocorrem grandes agregações de medusas (James \& Hermann 2001, James et al. 2005b, Benson et al. 2007a). Geralmente os indivíduos realizam movimentos mais localizados e restritos a pequenas áreas geográficas, sem uma direção aparente durante extensos períodos de tempo (desde semanas até vários meses) (James et al. 2005b, Benson et al. 2007b); alguns indivíduos já foram monitorados durante jornadas de ida e de volta ao longo de um ano emantiveram uma fidelidade regional em relação às áreas de alimentação (James et al. 2005b). Esta fidelidade regional também foi observada em fêmeas monitoradas desde as praias de desova no Mar do Caribe, quando algumas das fêmeas permaneceram na costa oeste do Atlântico norte, enquanto outras viajaram rumo ao norte e nordeste do Atlântico, e outras restringiram seus movimentos ao Atlântico tropical (Hays et al. 2006).

As agregações sazonais de medusas ocorrem frequentemente em áreas de ressurgências ou de descontinuidades físicas (ex. talude continental) (Graham et al. 2001), as quais são freqüentadas por indivíduos de D. coriacea (Eckert 2006, Eckert et al. 2006). Nos ambientes oceânicos $D$. coriacea se alimenta ao longo de frentes oceânicas, áreas de convergência, e outras feições oceanográficas, as quais provêem condições adequadas para a concentração de organismos gelatinosos. As tartarugas-de-couro têm sido observadas utilizando as águas produtivas associadas à corrente do Golfo, no Atlântico norte, e em sistemas dinâmicos de fortes correntes na passagem entre os oceanos Indico e Atlântico, ao sul do continente Africano. Nestas áreas, as tartarugas em geral alternam deslocamentos diretos a uma velocidade maior (favorecidos pelas fortes correntes) com traslados mais lentos circulares relacionados a feições oceanográficas específicas que sugerem a presença de comportamentos de alimentação (Luschi et al. 2006, Lambardi et al. 2008).

No Pacifico leste, as fêmeas que desovam na Costa Rica e México, após a migração através do corredor migratório, utilizam as águas oceânicas do Pacifico sul oriental como áreas de alimentação. Estas áreas são menos previsíveis em termos de produtividade (comparadas com as regiões costeiras perto do Peru e Chile no Pacifico leste) e, portanto, o comportamento de busca das manchas (patches) de presas por parte dos indivíduos é mais intenso (Shillinger et al. 2008, Reina et al. 2008). Essa diferença na produtividade primária líquida entre bacias oceânicas (por exemplo, entre o Pacífico e o Atlântico) permite explicar, em grande parte, as diferenças de tamanho e de sucesso reprodutivo (ex. produção total de ovos) das fêmeas. Assim, fêmeas maiores que amadureceram mais rápido depositam mais ovos do que fêmeas comparativamente menores (Saba et al. 2007).

\section{MOVIMENTOS MIGRATÓRIOS DESDE ÁREAS DE ALIMENTAÇÃO}

Até o momento, poucos transmissores colocados em fêmeas em áreas de desova têm transmitido por tempo suficiente para permitir que seja observado o comportamento das tartarugas durante seu retorno para estas áreas, especialmente porque o intervalo de remigração é geralmente igual ou superior a dois anos. Contudo, alguns estudos pioneiros na costa do Canadá, onde indivíduos de $D$. coriacea foram capturados no mar e monitorados via satélite, permitiram acompanhar os deslocamentos migratórios desde estas áreas de alimentação (James et al. 2005a,b,c, James et al. 2006b).

Durante os movimentos migratórios realizados desde as áreas temperadas de forrageamento locali- 
zadas na costa de Canadá em direção às áreas tropicais, as tartarugas-de-couro não transitam por rotas comuns e, em geral, utilizam extensas áreas do Atlântico norte. Apesar das variações existentes nas rotas individuais, em geral as tartarugas-de-couro que foram monitoradas durante migrações de ida e de volta entre áreas temperadas e tropicais apresentam uma certa fidelidade regional pelas áreas de alimentação (ex. voltam para a mesma região ocupada previamente) (James et al. 2005a). Geralmente o momento de partida das áreas de alimentação ocorre depois do verão boreal, durante os meses de outono. Nesta fase, a velocidade de navegação aumenta consideravelmente durante os primeiros dias ou nas primeiras semanas e se mantém relativamente elevada durante o período migratório (James et al. 2005b), sendo maior durante o período diurno do que durante o período noturno (Jonsen et al. 2006).

Indivíduos subadultos (comprimento curvilíneo da carapaça $(\mathrm{CCC})<140 \mathrm{~cm}$; James et al. 2006b) e adultos de ambos os sexos têm mostrado que realizam movimentos migratórios entre áreas temperadas e tropicais, inclusive durante anos não reprodutivos (ex. machos e fêmeas). A motivação para a realização de tais movimentos é ainda desconhecida, mas acredita-se que sirvam para aproveitar oportunidades de forrageamento sobre as agregações sazonais de presas durante o retorno às áreas de alimentação neríticas (James et al. 2005b).

\section{O SEXO E A CONDIÇÃO REPRODUTIVA AFETANDO OS PADRÕES COMPORTAMENTAIS NA MIGRAÇÃO}

O rastreamento de indivíduos nas áreas de alimentação oferece a oportunidade de examinar o comportamento de indivíduos de diferentes sexos e estágios reprodutivos. Assim, tem sido observado que os machos também realizam migrações para as áreas reprodutivas tropicais próximas às praias de desova localizadas no Mar do Caribe e na América Central, possivelmente em uma freqüência maior do que as fêmeas (James et al. 2005a,b,c). Além disto, algumas fêmeas, durante anos não reprodutivos, e alguns subadultos também costumam realizar migrações entre as áreas de alimentação na costa do Canadá e as áreas tropicais, embora tendam a permanecer em áreas oceânicas (longe da costa), em contraposição aos machos e as fêmeas durante os anos reprodutivos, os quais em geral se localizam em habitats costeiros (James et al. 2005b,c).

Embora a fidelidade por áreas de desova (ex. retorno para as mesmas áreas de desova durante sucessivas temporadas reprodutivas) tenha sido demonstrada para as fêmeas de $D$. coriacea (através da recaptura de indivíduos marcados), estudos de telemetria por satélite com machos desta espécie indicaram que estes também apresentam fidelidade por áreas de reprodução específicas (James et al. 2005c).

Uma diferença interessante entre os sexos é que durante os anos reprodutivos os machos tendem a chegar antes do começo da temporada de desova das fêmeas e tendem a se localizar em áreas próximas às praias de desova, ao contrário das fêmeas, que podem percorrer grandes distâncias no período entre uma desova e outra. Este comportamento tem sido visto como uma adaptação dos machos para maximizar as probabilidades de acasalamento com várias fêmeas antes que as primeiras desovas aconteçam (James et al. 2005c). Em geral, os machos iniciam a migração rumo às áreas de alimentação mais cedo do que as fêmeas, em período próximo ao mês de pico da temporada de desova (James et al. 2005c). Este padrão já foi observado em outras espécies de tartarugas marinhas, uma vez que as fêmeas de algumas espécies em geral rejeitam os machos após terem iniciado o período inicial de cópula (Hays et al. 2001) ou de oviposição (Plotkin et al. 1996).

\section{TENDÊNCIAS NA MOVIMENTAÇÃO VERTICAL}

Um importante aspecto das movimentações comportamentais das tartarugas-de-couro diz respeito às movimentações verticais por elas realizadas, as quais implicam em um considerável conjunto de ajustes fisiológicos, morfológicos e comportamentais que interagem para permitir e para tornar eficiente o mergulho em um amplo espectro de profundidades nos oceanos.

\section{OS MERGULHOS EM ÁREAS DE REPRODUÇÃO OU DESOVA}

Considerando que as características físicas dos ambientes marinhos próximos às praias de desova, em especial a batimetria, variam entre as regiões geográficas, os indivíduos de $D$. coriacea tendem a 
ajustar o seu comportamento de mergulho, de acordo com a batimetria do local (Georges et al. 2007).

A realização de mergulhos de maior profundidade e duração foram observados em indivíduos de D. coriacea em áreas de desova localizadas nas ilhas do Mar do Caribe, onde ambientes de águas profundas $(>1000 \mathrm{~m})$ ocorrem próximos à costa (Eckert 2002b, Eckert et al. 1989), em oposição aos mergulhos comparativamente mais rasos e de menor duração observados em áreas de desova continentais caracterizadas por extensas plataformas continentais (ex. Guiana Francesa) (Fosette et al. 2007).

Os mergulhos de maior duração de permanência no fundo (os chamados tipo-U), que indicam comportamentos de descanso ou de diminuição do gasto metabólico, foram observados em áreas do Pacífico oeste no Mar da China (Eckert et al. 1996) e no Pacifico leste na Costa Rica (Reina et al. 2005). Nessas áreas, as tartarugas permanecem em águas pouco profundas, realizando mergulhos de maior duração próximos ao fundo oceânico. Esse tipo de comportamento foi indicado como uma possível tentativa das fêmeas em evitar interações com os machos (Reina et al. 2005). A permanência delas por mais tempo no fundo oceânico restringiria a possibilidade das interações sexuais. Em contraste, no Atlântico, tanto nas áreas de desova localizadas em algumas ilhas do Mar do Caribe como na Guiana Francesa, as fêmeas de $D$. coriacea percorrem grandes distâncias e mergulham com acentuada freqüência, indicando que mantêm uma intensa atividade de movimentação entre uma e outra desova (Eckert 2002a, Fosette et al. 2007). Para fêmeas de $D$. coriacea na ilha Granada, no sul do Mar do Caribe foram observadas claras diferenças nos padrões de mergulho ao longo do ciclo diário, com a realização de mergulhos mais freqüentes e mais rasos durante o período noturno, sugerindo que as fêmeas em geral se alimentam de organismos gelatinosos que realizam migrações verticais (sobem em direção à superfície durante a noite e descem a maiores profundidades ao amanhecer) (Eckert et al. 1989, Myers \& Hays 2006). Embora na região da Guiana Francesa as fêmeas não possuam diferenças diárias nos padrões de mergulho, um tipo de mergulho (chamado como tipo-W), que é caracterizado por contínuas mudanças de profundidade ( $>2 \mathrm{~m}$ ) e por acentuados movimentos na fase profunda do mergulho, tem sido associado a possíveis eventos de alimentação (os quais foram inferidos a partir de registros de movimentos do bico, através de sensores mandibulares) (Fosette et al. 2008b).

De forma geral, as tartarugas-de-couro mostram uma tendência a realizar mergulhos mais profundos quanto maior for a distância que estejam da costa. Isto é sugestivo de que na maioria dos casos elas tendem a acompanhar a batimetria do fundo oceânico, principalmente nas áreas em que a plataforma continental é extensa (Fossette et al. 2007, Eckert et al. 1996). Tem sido mostrado que, próximo à costa, as tartarugas-de-couro costumam realizar mergulhos comparativamente mais rasos e com menor duração (o chamado mergulho sub-superficial ou do tipo-1). Este tipo de mergulho permite aos indivíduos se deslocarem de forma mais efetiva (ex. maior eficiência no nado, ver acima) na hora de abandonar as regiões do mar no entorno das praias de desova ou também em momentos próximos ao término do intervalo internidal, quando as tartarugas novamente se aproximam da costa (Southwood et al. 1999, Myer \& Hays 2006, Fosette et al. 2007).

O mergulho mais longo registrado até agora para D. coriacea foi de 86,5 min (López-Mendilaharsu et al. 2008), contudo, a maioria dos mergulhos apresenta uma duração 10-20 min (Hays et al. 2004, Sale et al. 2006). Vários autores têm calculado estimativas para o limite aeróbico do mergulho (ADL) (Lutcavage et al. 1992, Southwood et al. 1999, Wallace et al. 2005, Bradshaw et al. 2007) com a finalidade de avaliar os custos tempo-atividade para estes répteis marinhos (Wallace \& Jones 2008). Recentemente, utilizando medidas do gasto metabólico de indivíduos no mar, Wallace et al. (2005) estimaram esse limite entre 11,7 e 44,3 min, e Bradshaw et al. (2007), através de inferências do gasto metabólico de perfis de mergulho, reportaram que esse limite varia entre 19 e $48 \mathrm{~min}$. De acordo com Thompson \& Fedak (2001), os vertebrados marinhos de respiração aérea devem regularmente se aproximar dos limites do mergulho aeróbico (ADL) ou mesmo excedê-los, mesmo quando as condições de forrageamento forem vantajosas. Nas áreas de desova, as tartarugas-de-couro geralmente mergulham nesses limites, o que em alguns casos tem sido considerado como um indício adicional da ausência de alimentação (Wallace et al. 2005) e, em outros, como um comportamento de forrageamento oportunista embora não-ótimo (Fossette et al. 2007). 


\section{OS MERGULHOS EM HABITATS NERÍTICOS E EM HABITATS OCEÂNICOS}

Em ambientes oceânicos as tartarugas-de-couro são capazes de mergulhar até profundidades maiores do que $1000 \mathrm{~m}$ (Doyle et al. 2008, López-Mendilaharsu et al. 2008), mas a maioria dos mergulhos ocorre na zona epipelágica $(<200 \mathrm{~m})$ (Hays et al. 2004b). Em habitats neríticos, onde a batimetría restringe a profundidade máxima dos mergulhos, esta tartaruga se alimenta de organismos na coluna de água (James et al. 2006b). Portanto, as tartarugas-decouro podem modificar seus padrões de mergulho de forma oportunista, já que as presas geralmente se distribuem em manchas tanto em ambientes neríticos como oceânicos. Dermochelys coriacea apresenta então uma grande plasticidade comportamental para explorar áreas de forrageamento heterogêneas em termos de produtividade (Hays et al. 2006).

Um estudo realizado em filhotes e juvenis de $D$. coriacea (criados em cativeiro), onde foi monitorado o comportamento vertical e a resposta às presas nesses estágios de desenvolvimento, permitiu comprovar a existência de mergulhos tipo- $\mathrm{V}$ nos mesmos. Adicionalmente, os indivíduos maiores (7-10 semanas de vida) realizaram mergulhos mais profundos e de maior duração do que os indivíduos menores (2-6 semanas de vida), sendo que todos se alimentaram exclusivamente de organismos gelatinosos, indicando que os hábitos de vida de $D$. coriacea estão presentes após as primeiras semanas de vida dos filhotes (Salmon et al. 2004).

Nos adultos, o comportamento de movimentação vertical durante as migrações apresenta variações, dependendo do destino dos mesmos. Os indivíduos retornando para as áreas de alimentação imediatamente após a temporada de desova geralmente tendem a aproveitar as oportunidades de forrageamento ao longo da trajetória, modificando os padrões de mergulho e as suas atividades diárias de acordo com as condições locais (Hays et al. 2006). Deste modo as tartarugas passam a mergulhar continuamente e, geralmente, a duração dos mergulhos aumenta consideravelmente, sugerindo que isto se deva a uma maior taxa de encontro de presas (Hays et al. 2004a). Nesse período, os padrões de mergulho diários são evidentes e, em geral são caracterizados por mergulhos relativamente rasos e de maior duração durante a noite, em contraposição aos mergulhos profundos esporádicos e uma permanência de tempo predominante em águas superficiais durante o dia, sugerindo uma alimentação sobre organismos que realizam migrações verticais (Hays et al. 2004a, Hays et al. 2006, Eckert 2006, Sale et al. 2006) (ver acima).

Por outro lado, o comportamento vertical de $D$. coriacea tende a se modificar conspicuamente nas áreas de alimentação neríticas de regiões temperadas. Nessas áreas, os mergulhos tendem a ser mais rasos e mais breves e as diferenças nos padrões de mergulho entre períodos diurno e noturno tendem a desaparecer (James et al. 2006b). Geralmente nesses habitats as presas se concentram e se distribuem próximo à superfície, razão pela qual as tartarugas-de-couro não precisam mergulhar profundamente para localizar e capturar suas presas (James et al. 2006b, Hays et al. 2006, Eckert 2006). Este comportamento foi observado nas águas temperadas do Atlântico oeste, próximo à costa do Canadá (James et al. 2006b), em uma área de ressurgência em frente à costa da Mauritânia, na África (Eckert 2006) e ao sudoeste do continente Africano, na passagem do Oceano Indico para o Atlântico, em região sob a influência da corrente fria de Bengala (Sale et al. 2006). Em algumas áreas de alimentação da zona temperada (ex. costa este do Canadá) tem também sido observado que durante o período diurno as tartarugas-de-couro em geral permanecem mais tempo na superfície (Eckert 2006, James et al. 2006b). Isto é compatível com a observação de tartarugas manipulando as presas (James \& Herman 2001) e descansando na superfície (James et al. 2006b). Este comportamento observado em indivíduos de $D$. coriacea parece ter função em facilitar a digestão de grandes volumes de presas, as quais se encontram a baixas temperaturas, nas frias águas das regiões temperadas (James et al. 2005b, James et al. 2006b).

Em contraste ao comportamento dos indivíduos nas áreas temperadas de forrageamento, as tartarugasde-couro apresentam diferenças características nos padrões diários de mergulho durante as migrações para as áreas de reprodução e/ou de alimentação localizadas nos trópicos (James et al. 2006b). Estes padrões ao longo do ciclo diário de 24 horas (ex. maior proporção de tempo em águas superficiais durante o dia em relação à noite, mergulhos diurnos consideravelmente mais profundos e esporádicos em contraste aos 
mergulhos noturnos a menores profundidades e de longa duração) semelhantes aos observados durante as migrações pós-desova (ver acima) são consistentes com o comportamento de alimentação de $D$. coriacea sobre organismos que realizam migrações verticais (Hays et al. 2004a). Hipóteses alternativas para explicar estas variações nos padrões de mergulho também foram apresentadas por alguns autores, sendo que os indivíduos, após deixarem as áreas alimentação localizadas em altas latitudes, possivelmente apresentam uma menor necessidade de aproveitar as oportunidades de forrageamento durante a migração (James et al. 2005b, James et al. 2006b). Uma das hipóteses considera que os mergulhos profundos e de maior duração representam um comportamento para evitar possíveis predadores (ex. redução visual da silhueta na superfície). Contudo, se a predação nestas classes de tamanho (adulto e sub-adulto) é baixa, possivelmente existem vantagens adicionais que justifiquem passar esses longos períodos de tempo mergulhando. Adicionalmente, diferenças na flutuação dos indivíduos pela redução do tecido adiposo durante a migração, poderiam explicar as mudanças nos padrões de mergulho em relação às áreas de forrageamento (James et al. 2005b). Por outro lado, outras hipóteses indicam que os mergulhos profundos durante o dia podem ter uma função termorreguladora (ex. mergulhos profundos para esfriar o corpo durante períodos de elevada atividade) (James et al. 2005b) e que a maior proporção de tempo passado em águas superficiais durante o dia pode indicar oportunidades de receber indicações de navegação pelo sol durante a migração (James et al. 2006b).

\section{CONCLUSÕES}

Os estudos têm mostrado que as feições oceanográficas (sistemas de correntes, vórtices (eddies), frentes oceânicas e áreas de convergência, entre outros) são fatores-chave na determinação dos padrões de movimentação registrados em $D$. coriacea, produzindo movimentos de curso reto e constante em grande escala e trajetórias erráticas ou circulares em áreas pequenas. Em regiões caracterizadas por dinâmicas oceanográficas diferentes, as tartarugas-de-couro podem mostrar estratégias diversas de navegação, tanto aproveitando as correntes como nadando de forma independente a estas.
Existem diferenças comportamentais entre as colônias que desovam nas diversas regiões oceânicas. Por exemplo, encontramos tendências de ampla dispersão e movimentos migratórios em várias direções após o fim da temporada reprodutiva (ex. populações do Atlântico oeste e do Pacifico oeste) em contraposição com os movimentos migratórios pós-desova de populações onde os indivíduos se movimentam através de um corredor migratório comum (população do Pacífico leste). O destino final dessas migrações em alguns casos vai depender da disponibilidade de presas no período adequado nas diversas regiões oceânicas.

Apesar de que não esteja claro se $D$. coriacea se alimenta durante o período reprodutivo, existem dois tipos de comportamento bem diferenciados, onde a tendência de uma pouca movimentação para reduzir o gasto metabólico durante essa fase do ciclo migratório de algumas colônias (ex. Pacifico leste) se contrapõe à intensa movimentação com uma aparente busca de alimento por parte de outras (ex. Atlântico oeste).

As fêmeas que utilizamáreas de alimentação menos previsíveis onde o comportamento de busca é mais intenso (ex. Pacifico leste), geralmente apresentam um intervalo de remigração mais prolongado do que as fêmeas que se alimentam em regiões onde a disponibilidade de recursos é maior.

Em geral existe uma tendência ao predomínio de comportamentos de mergulho mais rasos nas áreas temperadas e comportamentos de mergulho profundo em águas tropicais e subtropicais. Estas mudanças latitudinais nos padrões de mergulho de $D$. coriacea parecem refletir mudanças na composição local e distribuição vertical das presas, uma vez que existe maior ocorrência de organismos gelatinosos de distribuição mais rasa em regiões de águas temperadas frias (altas latitudes) versus organismos de distribuição mais profunda em regiões de águas mais quentes (latitudes medias ou baixas) (Hays et al. 2006). Contudo, em regiões tropicais e subtropicais alguns fatores oceanográficos preponderantes (tais como as áreas de ressurgência, sistemas de correntes, frentes oceânicos, etc.) geralmente favorecem a concentração de organismos de distribuição mais rasa (ex. medusas), os quais podem provocar mudanças nos padrões de mergulho das tartarugas, fugindo em alguns casos, a essa tendência geral (Eckert 2006, Sale et al. 2006). 
A relativa falta de informação sobre populações ameaçadas nas áreas marinhas freqüentemente impede que estratégias adequadas de conservação sejam definidas. Entender os movimentos e os padrões de uso espacial de populações ameaçadas ao longo da sua área de distribuição constitui um elemento essencial para sua conservação, principalmente para espécies como as tartarugas marinhas, que podem utilizar amplas áreas oceânicas (Doyle et al. 2008). Neste sentido, o conhecimento sobre o comportamento referente a deslocamentos e o uso do habitat (tais como áreas de alimentação e de reprodução) podem auxiliar e orientar a definição de áreas prioritárias onde os esforços de conservação precisam ser mais efetivamente implementados (Luschi et al. 2006, Doyle et al. 2008).

AGRADECIMENTOS: Este estudo constitui parte da tese de Doutorado de M.L.M.. À Coordenação de Aperfeiçoamento de Pessoal de Nivel Superior (CAPES) pela bolsa de doutorado concedida a M.L.M. Ao Conselho Nacional de Desenvolvimento Científico e Tecnológico (CNPq) (processos n ${ }^{\circ} \mathrm{s}$. 307653/2003-0 e 477715/2006-0) e à FAPERJ (Fundação de Amparo à Pesquisa do Estado do Rio de Janeiro) através do Programa "Cientistas do Nosso Estado" (Processo E-26/100.471.2007) pelos auxílios concedidos a C.F.D.R.. A foto utilizada neste artigo foi gentilmente cedida pelo Banco de Imagens do Projeto TAMAR.

\section{REFERÊNCIAS}

BAILEY, H.; SHILLINGER, G.; PALACIOS, D.; BOGRAD, S.; SPOTILA, J.; PALADINO, F. \& BLOCK, B. 2008. Identifying and comparing phases of movement by leatherback turtles using state-space models. Journal of Experimental Marine Biology and Ecology, 356: 128-135.

BENSON, S.R.; DUTTON, P.H.; HITIPEUW, C.; SAMBER, B.; BAKARBESSY, J. \& PARKER, D. 2007a. Post-nesting migrations of leatherback turtles (Dermochelys coriacea) from Jamursba-Medi, Bird's Head Peninsula, Indonesia. Chelonian Conservation and Biology, 6: 150-154.

BENSON, S.R. KISOKAU, K.M.; AMBIO, L.; REI, V.; DUTTON, P.H. \& PARKER, D. 2007b. Beach use, internesting movement, and migration of leatherback turtles, Dermochelys coriacea, nesting on the north coast of Papua New Guinea. Chelonian Conservation and Biology, 6: 7-14.

BRODERICK, A.C.; GODLEY, B.J. \& HAYS, G.C. 2001. Trophic status drives inter-annual variability in nesting numbers of marine turtles. Proceedings of the Royal Society of London B, Biological Sciences, 268: 1481-1487.
CARR, A. 1952. Handbook of Turtles. Cornell University Press. Ithaca, NY. 542p.

CAUT, S.; GUIRLET, E.; ANGULO, E.; DAS, K. \& GIRONDOT, M. 2008. Isotope Analysis Reveals Foraging Area Dichotomy for Atlantic Leatherback Turtles. PLoS ONE, 3: e1845. doi:10.1371/ journal.pone.0001845.

DAVENPORT, J. 1998. Sustaining endothermy on a diet of cold jelly: energetics of the leatherback turtles Dermochelys coriacea. British Herpetological Society Bulletin, 62: 4-8.

DOYLE, T.K.; HOUGHTON, J.D.R.; O'SÚILLEABHÁIN, P.F.; HOBSON, V.J.; MARNELL, F.; DAVENPORT, J. \& HAYS, G.C. 2008. Leatherback turtles satellite-tagged in European waters. Endangered Species Research, 4: 23-31.

ECKERT, S.A. \& ECKERT, K.L. 1986. Harnessing leatherbacks. Marine Turtle Newsletter, 37: 1-3.

ECKERT, S.A \& SARTI, L.M. 1997. Distant fisheries implicated in the loss of the world's largest leatherback nesting population. Marine Turtle Newsletter, 78: 2-7.

ECKERT, S.A. 1998. Perspectives on the use of satellite telemetry and other electronic technologies for the study of marine turtles, with reference to the first year long tracking of leatherback sea turtles. In: S.P. Epperly \& J. Braun. (eds.). Proceedings of the Seventeenth Annual Symposium on Sea Turtle Biology and Conservation, NOAA Technical Memomorandum NMFSSEFSC-415. 294p.

ECKERT, S.A.; ECKERT, K.L.; PONGANIS, P. \& KOOYMAN, G.L. 1989. Diving and foraging behavior of leatherback sea turtles (Dermochelys coriacea). Canadian Journal of Zoology, 67: 2834-2840.

ECKERT, S.A.; LIEW, H.-C.; ECKERT.; K.L. \& CHAN, E.-H. 1996. Shallow water diving by leatherback turtles in the South China Sea. Chelonian Conservation and Biology, 2: 237-243.

ECKERT, S.A. 2002a. Swim speed and movement patterns of gravid leatherback sea turtles (Dermochelys coriacea) at St. Croix, U.S. Virgin Islands. Journal of Experimental Biology, 205: 3689-3697.

ECKERT, S.A. 2002b. Distribution of juvenile leatherback sea turtle Dermochelys coriacea sightings. Marine Ecology Progress Series, 230: 289-293.

ECKERT, S.A. 2006. High-use oceanic areas for Atlantic leatherback sea turtles (Dermochelys coriacea) as identified using satellite telemetered location and dive information. Marine Biology, 149: 1257-1267. 
ECKERT, S.A.; BAGLEY, D.; KUBIS, S.; EHRHART, L.; JOHNSON, C.; STEWART, K. \& DEFREESE, D. 2006. Internesting, post-nesting movements and foraging habitats of leatherback sea turtles (Dermochelys coriacea) nesting in Florida. Chelonian Conservation and Biology, 5: 239-248.

FERRAROLI, S.; GEORGES, J.-Y.; GASPAR, P. \& MAHO, Y.L. 2004. Where leatherback turtles meet fisheries. Nature, 429: 521-522.

FOSSETTE, S.; FERRAROLI, S.; TANAKA, T.; ROPERTCOUDERT, Y.; ARAI, N.; SATO, K.; NAITO, Y.; LE MAHO, Y. \& GEORGES, J.-Y. 2007. Dispersal and dive patterns in gravid leatherback turtles during the nesting season in French Guiana. Marine Ecology Progress Series, 338: 233-247.

FOSSETTE, S.; DE THOISY, B.; KELLE, L.; VERHAGE, B.; FRETEY, J. \& GEORGES, J.-Y. 2008a. The world's largest leatherback rookeries: conservation and research in French Guiana, Surinam and Gabon. Journal of Experimental Marine Biology and Ecology, 356: 69-82.

FOSSETTE, S.; GASPAR, P.; HANDRICH, Y.; LE MAHO, Y. \& GEORGES, J.-Y. 2008b. Dive and beak movement patterns in leatherback turtles (Dermochelys coriacea) during inter-nesting intervals in French Guiana. Journal of Animal Ecology, 77: 236-246.

FRETEY, J. 2001. Biogeography and Conservation of Marine Turtles of the Atlantic Coast of Africa. CMS Technical Series Publication no. 6, UNEP/CMS Secretariat, Bonn, Germany, 429 p.

GASPAR, P.; GEORGES, J.Y.; FOSSETTE, S.; LENOBLE, A.; FERRAROLI, S. \& MAHO, Y. 2006. Marine animal behaviour: neglecting ocean currents can lead us up the wrong track. Proceedings of the Royal Society B: Biological Sciences, 273: 2697-2702.

GEORGES, J.-Y. \& FOSSETTE, S. 2006. Estimating body mass in leatherback turtles Dermochelys coriacea. Marine Ecology Progress Series, 318: 255-262.

GEORGES, J.Y.; FOSSETTE, S.; BILLES, A.; FERRAROLI, S.; FRETEY, J.; GRÉMILLET, D.; LE MAHO, Y.; MYERS, A.E.; TANAKA, H. \& HAYS, G.C. 2007. Meta-analysis of movements in Atlantic leatherback turtles during the nesting season: conservation implications. Marine Ecology Progress Series, 338: 225-232.

GRAHAM, W.M.; PAGE`S, F. \& HAMNER, W.M. 2001. A physical context for gelatinous zooplankton aggregations: a review. Hydrobiologia, 451: 199-212.
HAYS, G.C.; BRODERICK, A.C.; GLEN, F.; GODLEY, B.J. \& NICHOLS, W.J. 2001. The movements and submergence behavior of male green turtles at Ascension Island. Marine Biology, 139: 395-399.

HAYS, G.C.; HOUGHTON, J.D.R.; ISAACS, C.; KING, R., LLOYD, C. \& LOVELL, P. 2004a. First records of oceanic dive profiles for leatherback turtles, Dermochelys coriacea, indicate behavioural plasticity associated with long-distance migration. Animal Behaviour, 67: 733-743.

HAYS, G.C.; HOUGHTON, J.D.R. \& MYERS, A.E. 2004b. PanAtlantic leatherback turtle movements. Nature, 429: 522-522.

HAYS, G.C.; HOBSON, V.J.; METCALFE, J.D.; RIGHTON, D. \& SIMS, D.W. 2006. Flexible foraging movements of leatherback turtles across the North Atlantic Ocean. Ecology, 87: 2647-2656.

HITIPEUW, C.; DUTTON, P.H.; BENSON, S.R.; THEBU, J. \& BAKARBESSY, J. 2007. Population status and internesting movement of leatherback turtles, Dermochelys coriacea, nesting on the northwest coast of Papua, Indonesia. Chelonian Conservation and Biology, 6: 28-36.

HOUGHTON, J.D.R.; DOYLE, T.K.; WILSON, M.W.; DAVENPORT, J. \& HAYS, G.C. 2006. Jellyfish aggregations and leatherback turtle foraging patterns in a temperate coastal environment. Ecology, 87: 1967-1972.

HUGHES, G.R.; LUSCHI, P.; MENCACCI, R. \& PAPI, F. 1998. The 7000-km oceanic journey of a leatherback turtle tracked by satellite. Journal of Experimental Marine Biology and Ecology, 229: 209-217.

JAMES, M.C. \& HERMAN, T.B. 2001. Feeding of Dermochelys coriacea on medusae in the northwest Atlantic. Chelonian Conservation and Biology, 4: 202-205.

JAMES, M.C. \& MROSOVSKY, N. 2004. Body temperatures of leatherback turtles Dermochelys coriacea in temperate waters off Nova Scotia, Canada. Canadian Journal of Zoology, 82: 13021306.

JAMES, M.C.; OTTENSMEYER, C.A. \& MYERS, R.A. 2005a. Identification of high-use habitat and threats to leatherback sea turtles in northern waters: new directions for conservation. Ecology Letters, 8: 195-201.

JAMES, M.C.; MYERS, R.A. \& OTTENSMEYER, C.A. 2005b. Behaviour of leatherback sea turtles, Dermochelys coriacea, during the migratory cycle. Proceedings of the Royal Society B: Biological Sciences, 272: 1547-1555.

JAMES, M.C.; ECKERT, S.C. \& MYERS, R.A. 2005 c. 
Migratory and reproductive movements of male leatherback turtles (Dermochelys coriacea). Marine Biology, 147: 845-853.

JAMES, M.C.; DAVENPORT, J. \& HAYS, G.C. 2006 a. Expanded thermal niche for a diving vertebrate : A leatherback turtle diving into near-freezing water. Journal of Experimental Marine Biology and Ecology, 355: 221-226.

JAMES，M.C.; OTTENSMEYER， C.A.; ECKERT，S.A. \& MYERS, R.A. 2006b. Changes in diel diving patterns accompany shifts between northern foraging and southward migration in leatherback turtles. Canadian Journal of Zoology, 84: 754-765.

JONSEN, I.D.; MYERS, R.A. \& JAMES, M.C. 2006. Robust hierarchical state-space models reveal diel variation in travel rates of migrating leatherback turtles. Journal of Animal Ecology, 75: 1046-1057.

KOOYMAN, G.L.; WAHRENBROCK, E.A.; CASTELLINI, M.A.; DAVIS, R.W. \& SINNETT, E.E. 1980. Aerobic and anaerobic metabolism during voluntary diving in Weddell seals: evidence of preferred pathways from blood chemistry and behavior. Journal of Comparative Physiology B, 138: 335-346.

LAMBARDI, P.; LUTJEHARMS, J.R.E.; MENCACCI, R.; HAYS, G.C. \& LUSCHI, P. 2008. Influence of ocean currents on long-distance movement of leatherback sea turtles in the Southwest Indian Ocean. Marine Ecology Progress Series, 353: 289-301.

LÓPEZ-MENDILAHARSU,M.; ROCHA,C.F.D.;DOMINGO, A.; WALLACE, B.P. \& MILLER, P. 2008. Prolonged, deep dives by the leatherback turtle Dermochelys coriacea: pushing their aerobic dive limits. JMBA2 - Biodiversity Records, 6274: 1-3.

LUSCHI, P.; SALE, A.; MENCACCI, R.; HUGHES, G.R.; LUTJEHARMS, J.R.E. \& PAPI, F. 2003. Current transport in leatherback sea turtles (Dermochelys coriacea) in the ocean Proceedings of the Royal Society B: Biological Sciences, 270(Suppl 2): 129-132.

LUSCHI, P.; LUTJEHARMS, J.R.E.; LAMBARDI, P.; MENCACCI, R.; HUGHES, G.R. \& HAYS, G.C. 2006. A review of migratory behaviour of sea turtles off southeastern Africa. South African Journal of Science, 102: 51-58.

LUTCAVAGE, M.E.; BUSHNELL, P.G. \& JONES, D.R. 1992. Oxygen stores and aerobic metabolism in the leatherback sea turtle. Canadian Journal of Zoology, 70: 348-351.

MARQUEZ, M.R. 1990. Sea turtles of the world. FAO Fisheries Synopsis No. 125, Vol. 11. Rome, FAO. 81p.

MEYLAN, A.B. 1982. Sea turtle migration - evidence from tag returns. Pp. 91-100. In: K.A. Bjorndal (ed.). Biology and Conservation of Sea Turtles. Smithsonian Institution Press, Washington DC. 583p.

MILLER, J.D. 1997. Reproduction in sea turtles. Pp. 51-81. In: P.L. Lutz \& J.A. Musick (eds.). The Biology of Sea Turtles, Vol. 1. CRC Press, Boca Raton, USA. 432p.

MORREALE, S.J.; STANDORA, E.A.; SPOTILA， J.R.; PALADINO, F.V. 1996. Migration corridor for sea turtles. Nature, 384: 319-320.

MUSICK, J.A. \& LIMPUS, C.J. 1997. Habitat utilization and migration in juvenile sea turtles. Pp. 137-163. In: P.L. Lutz \& J.A. Musick (eds.). The Biology of Sea Turtles, Vol. 1. CRC Press, Boca Raton, USA. 432p.

MYERS, A.E. \& HAYS, G.C. 2006. Do leatherback turtles Dermochelys coriacea forage during the breeding season? A combination of data-logging devices provide new insights. Marine Ecology Progress Series, 322: 259-267.

PLOTKIN, P.T.; OWENS, D.W.; BYLES, R.A. \& PATTERSON, R. 1996. Departure of male olive ridley turtles (Lepidochelys olivacea) from a nearshore breeding ground. Herpetologica, 52: 1-7.

PRITCHARD, P.C.H. 1973. International migrations of South American sea turtles (Cheloniidae and Dermochelyidae). Animal Behaviour, 21: 18-27.

PRITCHARD, P.C.H. 1976. Post-nesting movements of marine turtles (Chelonidae and Dermochelyidae) tagged in the Guianas. Copeia, 1976: 749-754.

REINA, R.D.; MAYOR, P.A.; SPOTILA, J.R.; PIEDRA, R. \& PALADINO, F.V. 2002. Nesting ecology of the leatherback turtle, Dermochelys coriacea, at Parque Nacional Marino Las Baulas, Costa Rica: 1988-89 to 1999-2000. Copeia, 2002: 653-664.

REINA, R.D.; ABERNATHY, K.J.; MARSHALL, G.J. \& SPOTILA, J.R. 2005. Respiratory frequency, dive behavior and social interactions of leatherback turtles, Dermochelys coriacea during the inter-nesting interval. Journal of Experimental Marine Biology and Ecology, 316: 1-16.

SABA, V.S.; SANTIDRIAN-TOMILLO, P.; REINA, R.D.; SPOTILA, J.R.; MUSICK, J.A.; EVANS, D.A. \&. PALADINO, F.V. 2007. The effect of the El Nino Southern Oscillation on the reproductive frequency of eastern Pacific leatherback turtles. Journal of Applied Ecology, 44: 395-404.

SABA, V.S.; SHILLINGER, G.L.; SWITHENBANK, A.M.; BLOCK, B.A.; SPOTILA, J.R.; MUSICK, J.A. \& PALADINO, F.V 2008 An oceanographic context for the foraging ecology of 
eastern Pacific leatherback turtles: Consequences of the ENSO. Deep-Sea Research I, 55: 646-660.

SALE, A.; LUSCHI, P.; MENCACCI, R.; LAMBARDI, P.; HUGHES, G.R.; HAYS, G.C.; BENVENUTI, S. \& PAPI, F. 2006. Long-term monitoring of leatherback turtle diving behaviour during oceanic movements. Journal of Experimental Marine Biology and Ecology, 328: 197-210.

SALMON, M.; JONES, T.T. \& HORCH, K.W. 2004. Ontogeny of Diving and Feeding behavior in Juvenile Seaturtles: Leatherback Seaturtles (Dermochelys coriacea L) and Green Seaturtles (Chelonia mydas) in the Florida Current. Journal of Herpetology, 38 (1): $36-43$.

SHILLINGER, G.L.; PALACIOS, D.M.; BAILEY, H.; BOGRAD, S.J.; SWITHENBANK, A.M.; GASPAR, P.; WALLACE, B.P.; SPOTILA, J.R.; PALADINO, F.V.; PIEDRA, R.; ECKERT, S.A. \& BLOCK, B.A. 2008. Persistent leatherback turtle migrations present opportunities for conservation. PLoS Biology, 6: e171 doi:10.1371/journal.pbio.0060171.

SOUTHWOOD, A.L.; ANDREWS, R.D.; LUTCAVAGE, M.E.; PALADINO, F.V.; WEST, N.H.; GEORGE, R.H. \& JONES, D.R. 1999. Heart rates and diving behavior of leatherback sea turtles in the Eastern Pacific Ocean. Journal of Experimental Biology, 202: 1115-1125.

SPOTILA,J.R.;DUNHAM,A.E.;LESLIE,A.J.;STEYERMARK, A.C.; PLOTKIN, P.T. \& PALADINO, F.V. 1996. Worldwide population decline of Dermochelys coriacea: Are leatherback turtles going extinct? Chelonian Conservation and Biology, 2: 209-222.

SPOTILA, J.R.; REINA, R.D.; STEYERMARK, A.C.; PLOTKIN, P.T. \& PALADINO, F.V. 2000. Biodiversity: Pacific leatherback turtles face extinction. Nature, 405: 529-530

THOMÉ, J.C.A.; BAPTISOTTE， C.; MOREIRA， L.M.; SCALFONI, J.T.; ALMEIDA, A.P.; RIETH DB. \& BARATA, P.C.R. 2007. Nesting Biology and Conservation of the Leatherback Sea Turtle Dermochelys coriacea in the State of Espírito Santo, Brazil, 1988-1989 to 2003-2004. Chelonian Conservation and Biology, 6: 15-27.

THOMPSON, D. \& FEDAK, M.A. 2001. How long should a dive last? A simple model of foraging decisions by breath-hold divers in a patchy environment. Animal Behaviour, 61: 287-296.

WALLACE, B.P.; WILLIAMS, C.L.; PALADINO, F.V.; MORREALE, S.J.; LINDSTROM, R.T. \& SPOTILA, J.R. 2005. Bioenergetics and diving activity of internesting leatherback turtles Dermochelys coriacea at Parque Nacional Marino Las Baulas, Costa Rica. Journal of Experimental Biology, 208: 3873-3884.
WALLACE, B.P. \& JONES, T.T. 2008. What makes sea turtles go: A review of metabolic rates and their consequences. Journal of Experimental Marine Biology and Ecology, 356: 8-24.

WITT, M.J.; BRODERICK, A.C.; JOHNS, D.J.; MARTIN, C.; PENROSE, R.; HOOGMOED, M.S. \& GODLEY, B.J. 2007. Prey landscapes help identify potential foraging habitats for leatherback turtles in the NE Atlantic. Marine Ecology Progress Series, 337: 231-244.

WITT, M.J.; BRODERICK, A.C.; COYNE, M.; FORMIA, A.; NGOUESSONO, S.; PARNELL, R.J.; SOUNGUET, G-P. \& GODLEY, B.J. 2008. Satellite tracking highlights difficulties in the design of effective protected areas for leatherback turtles during the internesting period. Oryx, 42(2): 296-300.

Submetido em 25/09/2008. Aceito em 27/11/2008. 